\title{
Dynamical transition in a nonlinear two-level system driven by a special hyperbolic-secant external field
}

\section{Hong Cao ( $\nabla$ ch198772ch@163.com )}

Chongqing Jiaotong University https://orcid.org/0000-0002-6797-3398

Xi-Jing Liu

Chongqing Jiaotong University

Miao Liu

Chongqing Jiaotong University

\section{Research Article}

Keywords: Exact solution model, Two-level system, Dynamical transition, Nonlinear effects

Posted Date: February 9th, 2022

DOI: https://doi.org/10.21203/rs.3.rs-1312397/v1

License: (c) (i) This work is licensed under a Creative Commons Attribution 4.0 International License.

Read Full License 


\title{
Dynamical transition in a nonlinear two-level system driven by a special hyperbolic-secant external field
}

\author{
Hong Cao · Xi-Jing Liu · Miao Liu
}

Received: date / Accepted: date

\begin{abstract}
We propose a simplification of the exact solution model in a linear system, which creates a transparent passage for dynamical transition. We extend this model to the nonlinear system and show that the transition dynamics will be determined by the nonlinear parameter and an unique parameter of external field. As non-linearity increases, Landau-Majorana-StückelbergZener (LMSZ) interference fringes can be constructive and the up energy level in the adiabatic limit splits into three levels. For fast-sweeping fields, we derive an analytic expression for dynamics transition under stationary phase approximation and show that transition probability will be blocked as the non-linearity is much larger than external field parameter, which agrees with numerical results.
\end{abstract}

Keywords Exact solution model, Two-level system, Dynamical transition, Nonlinear effects

\section{Introduction}

Exactly solvable quantum system with a single-axis driving field and corresponding dynamical behavior have long been a subject of considerable interest in quantum mechanics[1]. Among of them, the most famous example is the Landau-Majorana-Stückelberg-Zener (LMSZ) model with a linearly pulse[2,3], which remains a very active area of research due to numerous applications including quantum optics [4], solid-state physics [5], quantum information science[6] and a spin-orbit-coupled BoseEinstein condensate[7]. Another well known model is

H. Cao $(\varangle)$ - X.J.Liu · M.Liu

School of Material Science and Engineering, Chongqing Jiaotong University, Chongqing 400074, China

E-mail: ch198772ch@163.com the hyperbolic secant pulse of Rosen and Zener (RZ)[8], which has played an important role in ion-atom collision[9], self-induced transparency[10] and qubit control $[11,12]$. In contrast to LMSZ model where the energy bias is linearly varied in time driven by a $z$-axis control field, in RZ model, coupling strength is time dependent driven by a $x$-axis control field. Both of them belong to the family of analytical exact controls.

Recently, the increasing attention is focused on nonlinear quantum system because it exists in the ultracold Bose-Einstein condensate (BEC) system and nonlinear optics[13-17]. LMSZ and RZ model have also been extended to nonlinear case[18-22], in which the nonlinearity will dramatically affect the transition dynamics. Fox example, in nonlinear LMSZ model, there exists a critical value of the interaction strength beyond which the transition probability becomes nonzero even in the adiabatic limit. For nonlinear RZ model, the quantum transition can be completely blocked by a strong nonlinearity. Despite these isolated successes, extending exactly solvable single axis models to nonlinear cases have remained extremely rare. This is because that issues of instabilities and nonintegrability in nonlinear quantum system are expected to feature obstructions of control, it is difficult to apply the control principles of linear quantum system to nonlinear quantum system. Besides, analytically solvable model is rare, and these exact solutions of them expressed in terms of complicated special functions (such as the Gauss hypergeometric function and the Weber function), the overall time $(-\infty, \infty)$ will be required. To verify the correctness of numerical calculation, nonlinear case will also be entire time. The local time will dramatically affect dynamic result.

It is worth noting that a kind of exactly solvable single-axis driving model based on invariant method have been proposed, which can produce desired nonadi- 
abatic passages in two-level and three-level systems[2327]. Differing from original LMSZ and RZ model, these examples of analytically solvable system described by the simple solutions, rendering the control strategies more transparent. Therefore, numerical nonlinear result in these models will be simple to verify through a local time. These make us interesting to search for more exactly solvable single-axis driving models and examine what happens that dynamics affected by nonlinearity.

In this paper, we have constructed simple exact analytical solution for linear two-level system driven by a hyperbolic-secant pulse. It is note that our model is no redundant parameter and the driving field of the target Hamiltonian here is uniquely determined. Differing from the previous method which aims at finding out the solution of the special function, we resolve the dynamics of the system by simple solution. On the other hand, motivated by the recent increasing research for BEC, where nonlinearity naturally arises from a meanfield treatment of the interaction between particles. We extend our model to the nonlinear case and investigate its transition dynamics in the presence of nonlinearity. More interestingly, the influence of nonlinearity can be reduced by modulating a external parameter, so we can give a suitable values to get desirable quantum state control.

\section{Exact solutions for hyperbolic-secant model in linear two-level system}

We consider the nonlinear two-level system described by the dimensionless Schördinger equation (setting $\hbar=1$ ) $[17,18]$

$i \frac{d}{d t}\left(\begin{array}{l}a \\ b\end{array}\right)=H(t)\left(\begin{array}{l}a \\ b\end{array}\right)$,

with the Hamiltonian given by

$$
\begin{aligned}
H(t) & =\frac{\gamma}{2} \sigma_{x}+\left[\frac{\Omega_{z}(t)}{2}+\frac{\delta(t)}{2}\right] \sigma_{z}, \\
\delta(t) & =c\left(|a|^{2}-|b|^{2}\right),
\end{aligned}
$$

where $a$ and $b$ are the probability amplitudes, the total probability $|a|^{2}+|b|^{2}$ is conserved and set to be 1 . $c$ is the nonlinear parameter describing the interparticle interaction. $\sigma_{i=x, y, z}$ denote Pauli matrices satisfying $\left[\sigma_{i}, \sigma_{j}\right]=i \varepsilon_{i j k} \sigma_{k}, \gamma$ and $\Omega_{z}(t)$ are the coupling strength and the energy bias between two levels, which can be controlled by the external field (the magnetic field and the laser field), respectively. When $\mathrm{c}=0$ and $\gamma$ is constant, the Hamiltonian of equation (3) describes any linear two-level system which is driven along a single axis (denoted by $z$ )[1].
In present paper, we consider that the system is driven along a single axis $z$, so the coupling strength is constant and the energy bias is time dependent governed by a hyperbolic secant external field of the form

$\Omega_{z}(t)=2 \gamma \operatorname{sech}(\gamma \mathrm{t})$.

Here, we note that the external field is determined by an unique parameter, both the amplitude and scanning frequency can be replaced by $\gamma$, which is different from LMSZ and its nonlinear sweep model[18,28].

The above system can be exactly solved at the linear case $(c=0)$. To resolve the dynamic of this system, we suppose that the linear system possesses a dynamical invariant $[29,30]$

$I(t)=\frac{\sin \theta(t) \cos \varphi(t)}{2} \sigma_{x}+\frac{\sin \theta(t) \sin \varphi(t)}{2} \sigma_{y}+\frac{\cos \theta(t)}{2} \sigma_{z}$,

which satisfies

$\partial_{t} I(t)=-i[H(t), I(t)]$

Here, $\theta(t)$ and $\varphi(t)$ are auxiliary time-dependent angles. Eq.(5) is readily verified through the following equations of the components:

$$
\begin{aligned}
\gamma & =-\frac{\dot{\theta}(t)}{\sin \varphi(t)}, \\
\Omega_{z}(t) & =\dot{\varphi}(t)-\dot{\theta}(t) \cot \theta(t) \cot \varphi(t)-\delta(t) .
\end{aligned}
$$

According to Eqs.(6) and (7), we directly calculate the corresponding mixing angels $\theta(t)$ and $\varphi(t)$, which can be given by the following equations,

$\begin{aligned} \theta(t) & =\frac{\pi}{2}+\arccos (\tanh (\gamma t)), \\ \varphi(t) & =\pi-\arccos (\tanh (\gamma t)) .\end{aligned}$

We note that for given any $\theta(t)$ and $\varphi(t)$, one can construct $\gamma$ and $\Omega_{z}$. It is direct to verify that this system possesses a dynamical invariant

$I(t)=-\tanh (\gamma t)^{2} \frac{\sigma_{x}}{2}+\operatorname{sech}(\gamma \mathrm{t})^{2} \tanh (\gamma \mathrm{t}) \frac{\sigma_{\mathrm{y}}}{2}-\operatorname{sech}(\gamma \mathrm{t})^{2} \frac{\sigma_{\mathrm{z}}}{2}$.

Obviously, the instantaneous eigenstates of the invariant operator $I(t)$ in Eq. (9) read

$\left|\phi_{ \pm}\right\rangle= \pm \cos \frac{\theta}{2}| \pm\rangle+\sin \frac{\theta}{2} e^{ \pm i \varphi}|\mp\rangle$.

Here, for two-level system, we have used $|+\rangle=[1,0]^{T}$, $|-\rangle=[0,1]^{T}$. The dynamical basis of this system can be formulated as 

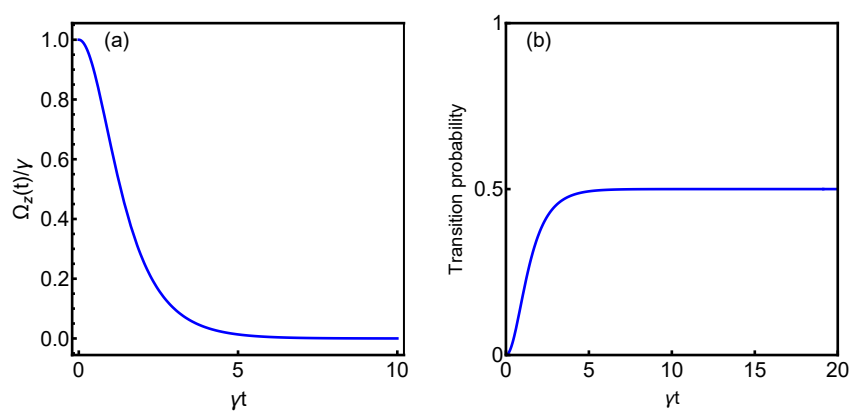

Fig. 1 The external field $\Omega_{z}(t)$ and transition probabilty(| $\left.a(t)\right|^{2}$ or $|b(t)|^{2}$ ) as time goes from 0 to $20 / \gamma$.

$$
|\Psi(t)\rangle=k_{1} e^{i \delta_{+}\left(t, t_{0}\right)}\left|\phi_{+}(t)\right\rangle+k_{2} e^{i \delta_{-}\left(t, t_{0}\right)}\left|\phi_{-}(t)\right\rangle,
$$

where $k_{1,2}$ are constant, the so-called lewis-Riesenfeld phase is given by

$$
\begin{aligned}
\delta_{ \pm}\left(t, t_{0}\right) & =\int_{t_{0}}^{t}\left\langle\phi_{ \pm}\left(t^{\prime}\right)\left|i \partial_{t^{\prime}}-H\left(t^{\prime}\right)\right| \phi_{ \pm}(t)\right\rangle d t^{\prime} \\
& =\mp \int_{t_{0}}^{t} \frac{\Omega_{x} \cos \varphi(t)}{\sin \theta(t)} d t .
\end{aligned}
$$

So far, we have given the exact solution of linear system. Consequently, the probability amplitudes can be generically expressed as $a=\langle+\mid \Psi(t)\rangle$ and $b=\langle-\mid \Psi(t)\rangle$.

In the following study, we assume the quantum state is prepared on one mode initially. As the external field turned on, quantum transition between two modes occur. In realistic system, the truncation of driving field is inevitable, so we consider that the local time evolve from 0 to $\frac{20}{\gamma}$. If the system starts from $|+\rangle$, then the time-dependent mode superposition coefficients $\left(k_{1,2}\right)$ are determined by $k_{1,2}=\langle \pm \mid \Psi(0)\rangle$, it yields $k_{1}=0$ and $k_{2}=1$, and the transition probability is given by $P(t)=|b(t)|^{2}=\cos ^{2} \frac{\theta}{2}$. On the other hand, if the system starts from $|-\rangle$, then the superposition coefficients $\left(k_{1,2}\right)$ satisfy $k_{1}=1$ and $k_{2}=0$, the transition probability can also be expressed as $P(t)=|a(t)|^{2}=$ $\cos ^{2} \frac{\theta}{2}$. It is clear that each of them are sysmmetry. This result provides a transparent control in linear system. We depict the external field format and transition probability in Fig.1.

\section{Nonlinearity effects}

\subsection{Transition dynamics in nolinear two-level system}

With the emergence of nonlinearity $(c \neq 0)$, the transition dynamics will dramatically change[31]. The Schrödinger Eq.(1) is no longer analytically solvable, we therefore exploit a 4-5th-order Runge-Kutta algorithm to trace the quantum state evolution numerically, the contour plots for transition probability with different initial states have been shown in Fig.2. In our calculation, the transition probability as the function of both time (evolving from 0 to $20 / \gamma$ ) and $c / \gamma$. As shown in Fig.2, the bule region stands for low transition probability, and red area corresponds to high transition probability.

In linear case $(c / \gamma=0)$, it is shown that the transition dynamics are symmetrical when the system starts from the state $|+\rangle$ and $|-\rangle$, which is robustness and quickly stabilized 0.5 , this result agrees with the analytic prediction of Fig.1. However, the symmetry will be broken as the nonlinear interaction increases. This phenomena is similar to the references[17,21]. Differing from them, in this model, when the scanning frequency is fast enough, the transition dynamics will be close to linear case, which can be strongly modified by the scanning frequency. Meanwhile, as the value of $c / \gamma$ increases, LMSZ interferometry fringes can be constructive, even if the driving field is not periodic variety. However, the LMSZ interference patterns are not symmetry in Fig.2(a) and Fig.2(b). These effects provide good opportunity to precisely measure the interaction between interferometry phenomena and external control fields.

For the weak nonlinear case, i.e., $c / \gamma \leq 1$, the oscillation behavior gradually appears no matter what the initial state $|+\rangle$ or $|-\rangle$ is, and the maximum value of transition probability is even larger than 0.5 in some times. However, the oscillation period of two patterns are different. When the system is prepared on state $|+\rangle$ (see Fig.2(a)), it will decrease as the nonlinearity strengths. Starting from state $|-\rangle$ exhibits the opposite behavior(see Fig.2(b)). For the strong interaction, i.e., $c / \gamma>1$, quantum transition also exhibits striking difference between preparing on $|+\rangle$ (see Fig.2(a)) and starting from $|-\rangle$. When the system is initially prepare on state $|+\rangle$, the oscillation behavior even appears in $c / \gamma>2$, and the oscillation pattern is completely broken at $c / \gamma=4$, both the oscillation period and amplitude have a big change at this case. The quantum transition probability between two modes is blocked and close to zero. When the system is initially prepare on state $|-\rangle$ (see Fig.2(b)), the amplitude of oscillation pattern is dramatically reduced at $c / \gamma=2$, the transition probability is block and tends to zero at this case. Obviously, starting from $|+\rangle$ needs to stronger nonlinearity to make the transition probability block.

These results show that there exist a critical value of $c / \gamma$, the transition probability will be dramatically reduced. The LMSZ interference patterns can be destructive or constructive, which are determined by $c / \gamma$. 

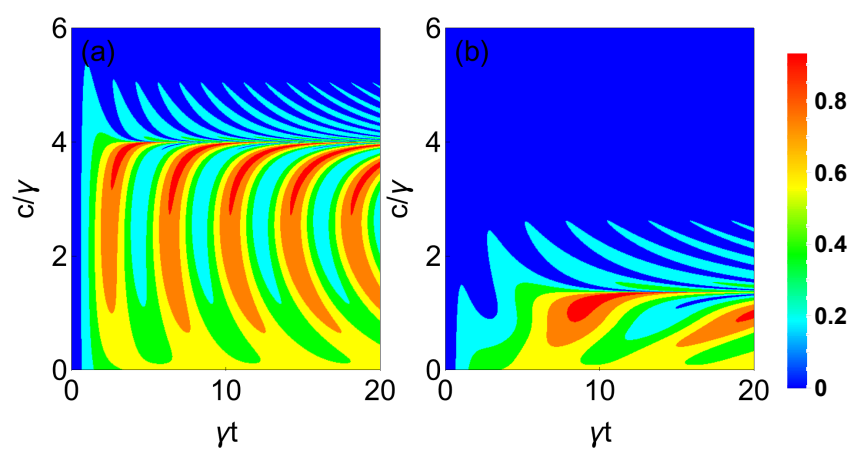

Fig. 2 Contour plot of transition probability as the function of time $(\gamma t)$ and the ratio of nonlinear parameter $c$ to scaning frequency $\gamma$ with different initial state. (a)the system starts from $|+\rangle$, (b)the system is prepared on $|-\rangle$.
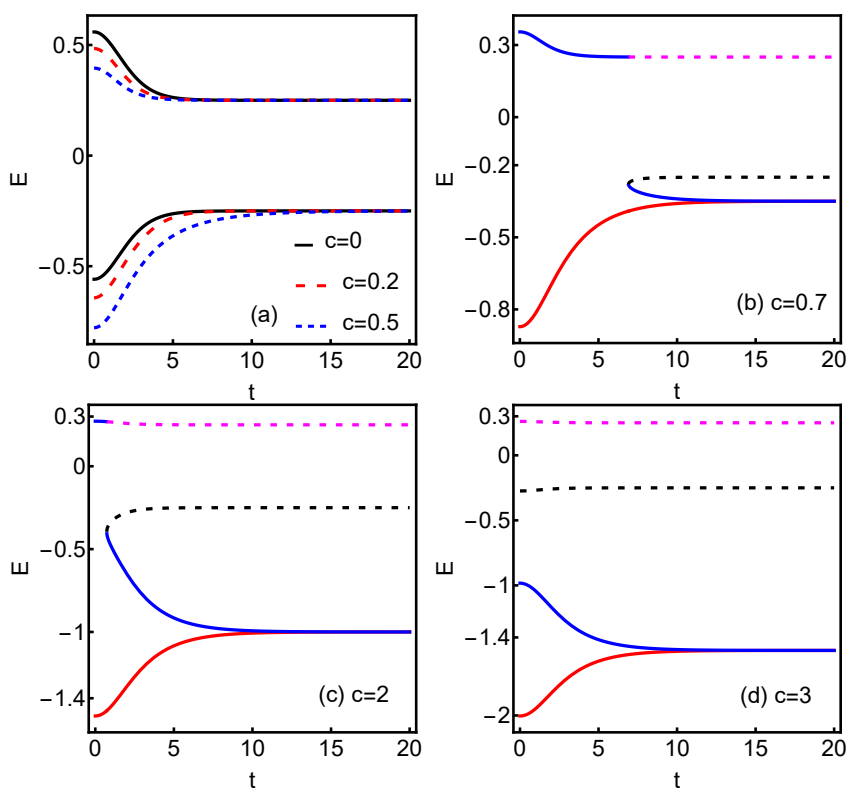

Fig. 3 The adiabatic energy-levels at $\gamma=0.5$ :(a)linear case $(c=0)$, (b)weak nonlinear case $(c=0.5)$, (c)strong nonlinear case $(c=2)$, (d)strong nonlinear case $(c=3)$. The dashed lines in (b),(c),(d) correspond to unstable energy level.

That is to say, if the system is initially prepare on state $|+\rangle$, this point is at $c / \gamma=4$. Starting from $|-\rangle$ is at $c / \gamma=1.4$. As a result, we can modulate the value of $c / \gamma$ to get desirable transition probability or LMSZ interference patterns. In the following, let us explicitly consider the influence of non-linearity effects at the case of low or fast scanning frequency.

\subsection{Adiabatic limit}

In the adiabatic limit, the scanning frequency $(\gamma)$ is very slow and the characters of transition probabilities should be entirely determined by adiabatic energy levels and eigenstates[32]. These levels as the solution of the time-dependent version of Eq.(1) obtained by replacing $i(\partial / \partial t)$ with energy $E$, after some elaboration, we find the following quartic equation

$(2 E+c)^{2}\left(4 E^{2}-\gamma^{2}\right)-4 \Omega_{z}^{2} E^{2}=0$

The adiabatic energy levels of system are given by the roots of above quartic equation. We illustrate these levels with $\gamma=0.5$ in Fig.3 for the case of linearity, weak nonlinearity, strong nonlinearity, respectively. It is shown that the levels will be dramatically affected by the increasing nonlinearity. In the linear case (see Fig.3(a)), two energy levels are symmetric about a horizontal axis, which leads to symmetrical transition dynamics. However, the symmetry will be broken by the presence of nonlinearity.

For the case of weak nonlinearity, there exists two situations, one case is that the number of levels are two at $c \leq 0.5$, i.e., $c / \gamma \leq 1$, which is similar to its linear counterpart. Compared with the linearity case, we see that the energy levels are lower than the ones for $c=0$ (see Fig3.(a)), and it leads to a oscillation behavior of transition probability in Fig.2 (the case of $c / \gamma<1$ ). Another case is that the number of levels are four at $c=0.7$, i.e., $c / \gamma=1.4$ (see Fig.3(b)). In Fig.3(b), the dashed levels are unstable, which can be evaluated by Eq.(15). The eigenvalues $E$ can be real, complex, or pure imaginary. Only pure imaginary eigenvalues correspond to stable states; Others indicate the unstable ones. We note that it is different from the reference[18, 19], there is no looping new feature in our figures. As is shown in Fig.3(b), the up level divides into three levels, which include two unstable levels and one stable levels, the breakdown of adiabatic evolution even in the adiabatic limit. However, the lowest level do not split. They are also in good agreement with the situation results in Fig.2. When the system is prepared initially in state $|+\rangle$, the system follows the up-level until level structure destroyed, it only presences the low transition probability and oscillation behavior. As the nonlinearity increases, the up-level splits and the transition probability becomes larger. Owing to the up-level do not completely transform into the unstable level, the transition probability of starting from $|+\rangle$ escapes to be blocked. When the system is prepared initially in state $|-\rangle$, the system follows the lowest-level until the up-level structure is destroyed, which leads to no way to jump to the up level, because the middle level and the lowest-level degenerate. As a result, transition probability block and closes to 0 at $c>0.7(c / \gamma>1.4)$.

For the case of strong nonlinearity $(c>1)$, i.e, Fig3(c) and Fig3(d), there also exists four levels. In Fig.3(c), we note that the up level gradually turn to unstable level and the middle two levels close to the right vertical 

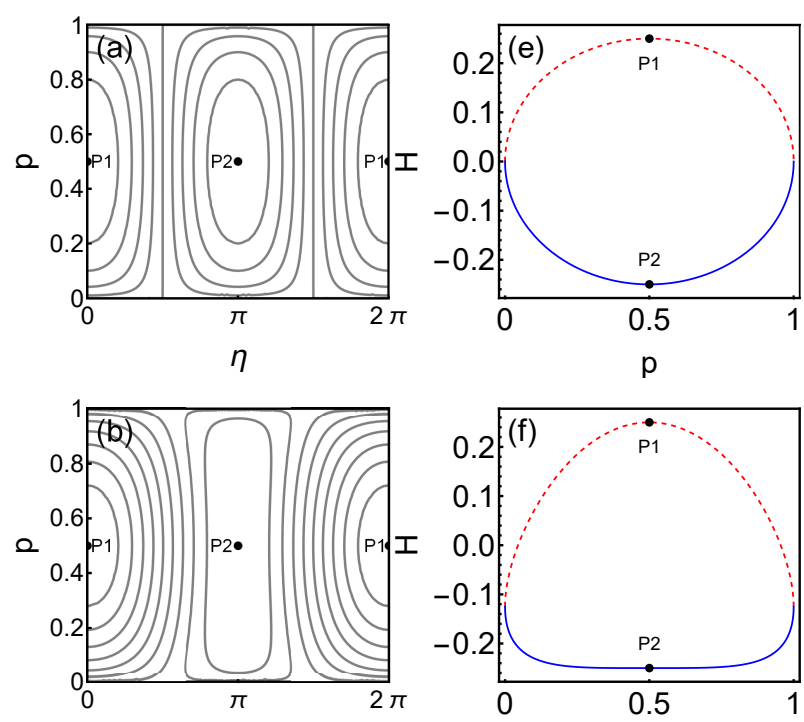

$\eta$
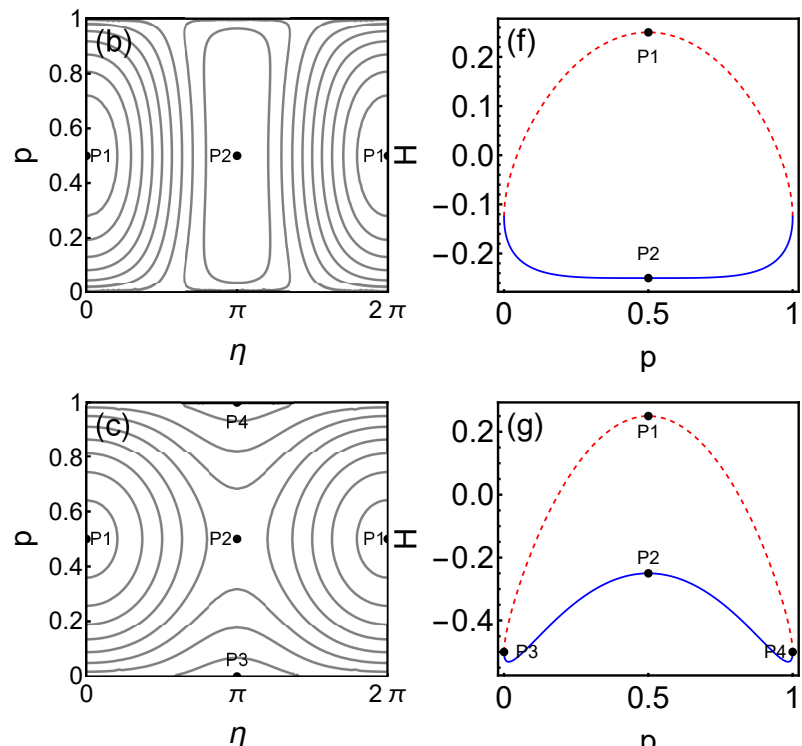

$\mathrm{p}$
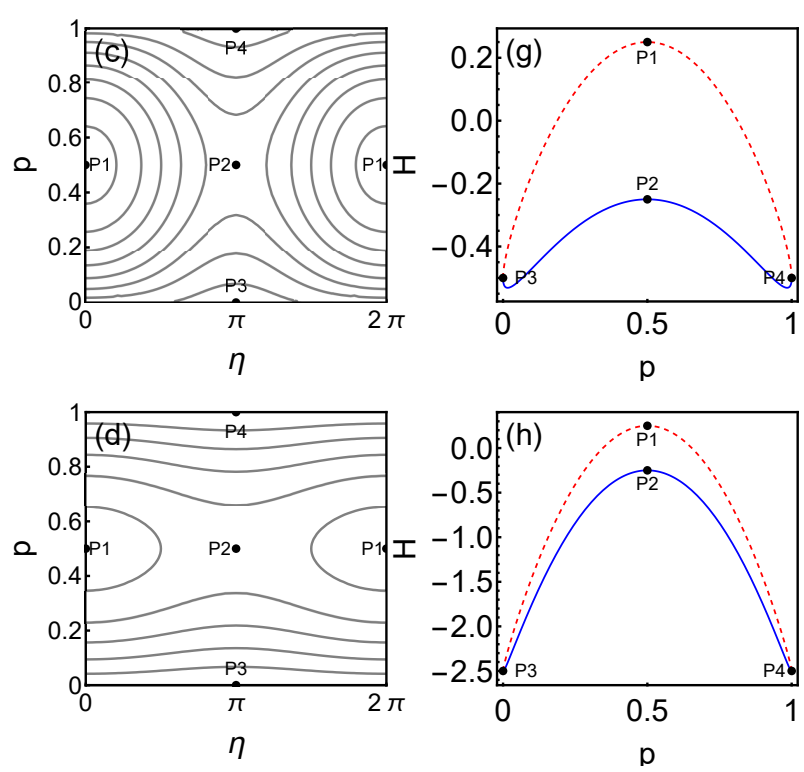

Fig. 4 Evolution of the phase space motions as as $c$ changes $(\mathrm{a}) \mathrm{c}=0,(\mathrm{~b}) \mathrm{c}=0.5,(\mathrm{c}) \mathrm{c}=2(\mathrm{~d}) \mathrm{c}=3$. The second column is the corresponding energy curve at $\eta=0$ (dashed line) and $\eta=0$ (heavy line).

axis at $c \leq 2$,i.e., $c / \gamma=4$. The transition probability also exists oscillation behavior. However, when the nonlinearity effect is enough strong (see Fig.3(d)),i.e., $c>2$, four levels will be completely formed, which is not changed as the nonlinearity parameter $c$ increases and the transition probability will be blocked. These results perfectly agree with Fig.(2).

To further explore the above peculiar phenomena, we construct the effective classical Hamiltonian introducing the transition probability $P=|b(t)|^{2}$ and $\eta=$ $\eta_{b}-\eta_{a}$ as the relative phase of two modes[32], then we obtain an effective Hamiltonian and satisfy the canon-

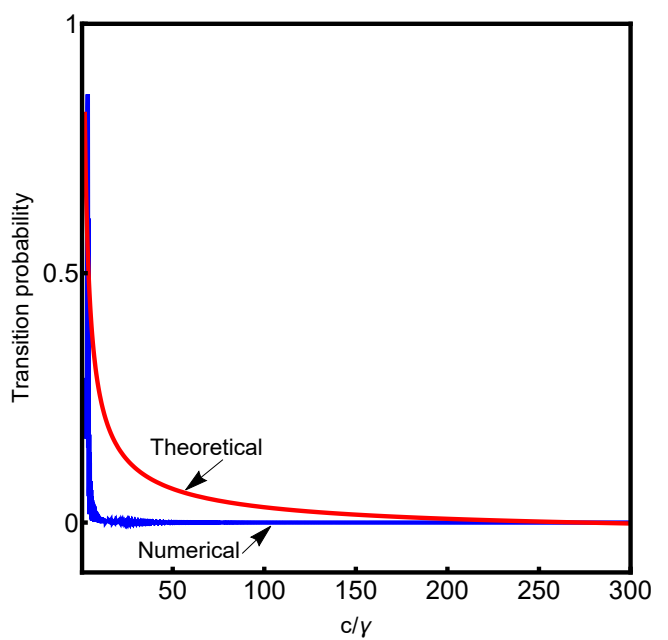

Fig. 5 Comparison between our analytic result using SPA and the numerical integration of Schrödinger equation (1).

ical equation,i.e., $\dot{p}=-\partial H / \partial \theta, \dot{\theta}=\partial H / \partial p$,

$H(t)=\frac{\Omega_{z}}{2}(2 p-1)-\frac{c}{4}(2 p-1)^{2}+\Omega_{x} \sqrt{p(1-p)} \cos \eta$,

which can completely describe the dynamical properties of system(1). Its fixed points correspond to the eigenstates of the nonlinear two-level system, which can be given by the following equations:

$\eta^{*}=0, \pi$

$\Omega_{z}-c\left(2 p^{*}-1\right)+\frac{\Omega_{x}\left(2 p^{*}-1\right)}{2 \sqrt{p^{*}\left(1-p^{*}\right)}} \cos \eta^{*}=0$,

$\Omega_{x} \sqrt{p^{*}\left(1-p^{*}\right)} \sin \eta^{*}=0$.

We have depicted the phase space orbits of the corresponding system (14) in Fig.4 with the time $t=$ 20 , there exists two or four the fixed points (labeled $P 1, P 2, P 3, P 4)$. The number of fixed points depend on the nonlinear parameter $c$. For weak nonlinearity, $c<1$, there exists only two fixed points ( $P 1$ and $P 2$ ) in Fig.4(a) and Fig.4(b)). They are elliptic points, each being surrounded by closed (elliptic) orbits, which are located on $\theta^{*}=0$ and $\theta^{*}=\pi$, and meaning that the two corresponding eigenstates of the two-level system have relative phase of $\pi$. The stable elliptic fixed point $P 1$ corresponds to the lower level in Fig.3. $P 2$ is the upper level. For weak nonlinearity, $c>1$, when we increase $c, P 2$ bifurcates into $P 3$ and $P 4$ (see Fig.4(c) and Fig.4(d)), the corresponding up level split into two unstable level in Fig.3(c) and Fig.3(d). 


\subsection{Sudden limit}

In this section, we will discuss quantum transition in sudden limit, and the scanning frequency is fast enough. We can derive the analytical expression of the transition probabilities using the stationary phase approximation (SPA). Here, we focus on the case of strong nonlinearity $c / \gamma \gg 1$. We thus expect the amplitude $b$ remains small and $a \sim 1$ all the time, and a perturbation treatment of the problem becomes adequate.

We begin with the variable transformation,

$a=a^{\prime} \exp \left[-\mathrm{i} \int_{0}^{\mathrm{t}}\left(\frac{\Omega_{\mathrm{z}}}{2}\right)+\frac{\mathrm{c}}{2}\left(|\mathrm{~b}|^{2}-|\mathrm{a}|^{2}\right)\right] \mathrm{dt}$,

$b=b^{\prime} \exp \left[-\mathrm{i} \int_{0}^{\mathrm{t}}\left(\frac{\Omega_{\mathrm{z}}}{2}\right)+\frac{\mathrm{c}}{2}\left(|\mathrm{~b}|^{2}-|\mathrm{a}|^{2}\right)\right] \mathrm{dt}$.

As a result, the diagonal terms in Hamiltonian are transformed away, and we have

$b^{\prime}=\frac{\gamma}{2 i} \int_{0}^{t} d t \exp \left(-\mathrm{i} \int_{0}^{\mathrm{t}}\left[\Omega_{\mathrm{z}}+\mathrm{c}\left(|\mathrm{b}|^{2}-|\mathrm{a}|^{2}\right)\right] \mathrm{dt}\right)$

Following Ref.[19], we evaluate the self-consistently of Eq.(20). Owing to the large $\gamma$, the integrand gives a rapid phase oscillation, and it makes the integral small. The dominant contribution comes from the stationary $t_{0}$ of the phase around, and we have

$\Omega_{z}+c\left(2|b|^{2}-1\right)=\bar{\alpha}\left(t-t_{0}\right)$

where

$\bar{\alpha}=\left(\frac{d \Omega_{z}}{d t}+2 c \frac{d|b|^{2}}{d t}\right)_{t_{0}}$

Since $|b(t)|^{2}=\left|b^{\prime}(t)\right|^{2}$, then we have

$|b|^{2}=\left(\frac{\gamma}{2}\right)^{2} \int_{0}^{t} d t\left|\exp \left(-\frac{\mathrm{i}}{2} \bar{\alpha}\left(\mathrm{t}-\mathrm{t}_{0}\right)^{2}\right)\right|^{2}$

Combining the relation (22) with Eq.(23), we come to a closed equation for $\bar{\alpha}$,

$\bar{\alpha}=-2 \gamma^{2} \operatorname{sech}\left[\gamma \mathrm{t}_{0}\right] \tanh \left[\gamma \mathrm{t}_{0}\right]+2 \mathrm{c}\left(\frac{\gamma}{2}\right)^{2} \sqrt{\frac{2 \pi}{\bar{\alpha}}}$

Here, we have differentiated Eqs.(23) at time $t_{0}$, obtaining a few standard Fresnel integrals with the result $[(d \mid$ $\left.\left.\left.b\right|^{2} / d t\right)\right]_{t_{0}}=(\gamma / 2)^{2} \sqrt{2 \pi / \bar{\alpha}}$, and the nonadiabatic transition probability described by

$\Gamma=\frac{\pi \gamma^{2}}{2 \bar{\alpha}}$

Then the above result yields a closed equation for $\Gamma$,

$\frac{1}{\Gamma}=-\frac{4}{\pi} \operatorname{sech}\left[\gamma \mathrm{t}_{0}\right] \tanh \left[\gamma \mathrm{t}_{0}\right]+\frac{2 \mathrm{c}}{\pi \gamma} \sqrt{\Gamma}$
The equation (26) gives the approximate solution of transition probability $|b(t)|^{2}$ in the sudden limit. Compared with numerical result, it shows good agreement at $c / \gamma>200, c / \gamma<200$ a clear deviation is observable in Fig.5. It is due to the the enough fast of scanning frequency leads to the invalidity of our assumption $a \sim 1$. Consequently, when $c \gg \gamma$, transition probability will be blocked.

\section{Conclusion}

In conclusion, we have constructed a single-axis driving model and given a simple analytical solution for linear two-level system. For nonlinear case, we investigated dynamics transition of this model, which is influenced by the scanning frequency $(\gamma)$ and nonlinear parameter (c). There exists a critical value for $c / \gamma$, LMSZ interference fringes will be destructive at this point. On the other hand, the adiabatic energy level will be splitted and one of fixed point bifurcates into two points as nonlinearity increases. When the scanning frequency is fast enough, we derive an analytic result for dynamic transition and show that we need more large nonlinearity value to make the transition probability close to zero.

\section{Data Availability Statements}

All data generated or analyzed during this study are included in this article.

\section{Conflict of interest}

There is no conflict of interest.

\section{References}

1. Barnes, E., Sarma, S.D.: Analytically solvable driven time-dependent two-level quantum systems. Physical review letters 109(6), 060401 (2012)

2. Landau, L.: On the theory of transfer of energy at collisions ii. Phys. Z. Sowjetunion 2(46), 118 (1932)

3. Zener, C.: Non-adiabatic crossing of energy levels. Proceedings of the Royal Society of London. Series A, Containing Papers of a Mathematical and Physical Character $\mathbf{1 3 7}(833)$, 696-702 (1932)

4. Bouwmeester, D., Dekker, N., Dorsselaer, F.v., Schrama C., Visser, P., Woerdman, J.: Observation of landauzener dynamics in classical optical systems. Physical Review A 51(1), 646 (1995)

5. Wernsdorfer, W., Sessoli, R., Caneschi, A., Gatteschi, D., Cornia, A.: Nonadiabatic landau-zener tunneling in fe8 molecular nanomagnets. EPL (Europhysics Letters) $\mathbf{5 0}(4), 552(2000)$ 
6. Fuchs, G., Burkard, G., Klimov, P., Awschalom, D.: A quantum memory intrinsic to single nitrogen-vacancy centres in diamond. Nature Physics 7(10), 789-793 (2011)

7. Olson, A.J., Wang, S.J., Niffenegger, R.J., Li, C.H., Greene, C.H., Chen, Y.P.: Tunable landau-zener transitions in a spin-orbit-coupled bose-einstein condensate. Physical Review A 90(1), 013616 (2014)

8. Rosen, N., Zener, C.: Double stern-gerlach experiment and related collision phenomena. Physical Review 40(4), $502(1932)$

9. Olson, R.E.: Charge transfer at large internuclear distances: application to asymmetric alkali-ion-alkali-atom systems. Physical Review A 6(5), 1822 (1972)

10. McCall, S.L., Hahn, E.L.: Self-induced transparency. Physical Review 183(2), 457 (1969)

11. Suominen, K.A., Garraway, B.M., Stenholm, S.: Wavepacket model for excitation by ultrashort pulses. Physical Review A 45(5), 3060 (1992)

12. Poem, E., Kenneth, O., Kodriano, Y., Benny, Y., Khatsevich, S., Avron, J., Gershoni, D.: Optically induced rotation of an exciton spin in a semiconductor quantum dot. Physical review letters 107(8), 087401 (2011)

13. Morsch, O., Oberthaler, M.: Dynamics of bose-einstein condensates in optical lattices. Reviews of modern physics 78(1), 179 (2006)

14. Dou, F.Q., Cao, H., Liu, J., Fu, L.B.: High-fidelity composite adiabatic passage in nonlinear two-level systems. Physical Review A 93(4), 043419 (2016)

15. Zhu, J.J., Chen, X., Jauslin, H.R., Guérin, S.: Robust control of unstable nonlinear quantum systems. Physical Review A 102(5), 052203 (2020)

16. Lahini, Y., Pozzi, F., Sorel, M., Morandotti, R., Christodoulides, D.N., Silberberg, Y.: Effect of nonlinearity on adiabatic evolution of light. Physical review letters 101(19), 193901 (2008)

17. Feng, P., Wang, W.Y., Sun, J.A., Dou, F.Q.: Demkovkunike transition dynamics in a nonlinear two-level system. Nonlinear Dynamics 91(4), 2477-2484 (2018)

18. Liu, J., Fu, L., Ou, B.Y., Chen, S.G., Choi, D.I., Wu, B., Niu, Q.: Theory of nonlinear landau-zener tunneling. Physical Review A 66(2), 023404 (2002)

19. Wang, G.F., Ye, D.F., Fu, L.B., Chen, X.Z., Liu, J.: Landau-zener tunneling in a nonlinear three-level system. Physical Review A 74(3), 033414 (2006)

20. Li, S.C., Fu, L.B., Liu, J.: Nonlinear landau-zenerstückelberg-majorana interferometry. Physical Review A 98(1), 013601 (2018)

21. Ye, D.F., Fu, L.B., Liu, J.: Rosen-zener transition in a nonlinear two-level system. Physical Review A 77(1), $013402(2008)$

22. Li, S.C., Fu, L.B.: Nonlinear rosen-zener-stückelberg interferometry of a bose-einstein condensate. Physical Review A 102(3), 033313 (2020)

23. Li, W., Cen, L.X.: Coherent population transfer in multilevel allen-eberly models. Quantum Information Processing 17(4), 1-13 (2018)

24. Zhao, P.J., Li, W., Cao, H., Yao, S.W., Cen, L.X.: Exotic dynamical evolution in a secant-pulse-driven quantum system. Physical Review A 98(2), 022136 (2018)

25. Cao, H., Yao, S.W., Cen, L.X.: Explicit construction of nonadiabatic passages for stimulated raman transitions. Physical Review A 100(5), 053410 (2019)

26. Cao, H., Yao, S.W., Cen, L.X.: Anomalous dynamical evolution and nonadiabatic level crossing in exactly solvable time-dependent quantum systems. The European Physical Journal D 73(10), 1-5 (2019)
27. Cao, H., Zhao, R.Q., Chen, H.: Accurate theoretical analysis of light transition in three-waveguide directional coupler based on invariant engineering. Optical and Quantum Electronics 53(6), 1-10 (2021)

28. Dou, F.Q., Li, S.C., Cao, H.: Combined effects of particle interaction and nonlinear sweep on landau-zener transition. Physics Letters A 376(1), 51-55 (2011)

29. Lewis Jr, H.R.: Classical and quantum systems with time-dependent harmonic-oscillator-type hamiltonians. Physical Review Letters 18(13), 510 (1967)

30. Lewis Jr, H.R., Riesenfeld, W.: An exact quantum theory of the time-dependent harmonic oscillator and of a charged particle in a time-dependent electromagnetic field. Journal of mathematical physics 10(8), 1458-1473 (1969)

31. Wu, Y., Yang, X.: Strong-coupling theory of periodically driven two-level systems. Physical review letters 98(1), 013601 (2007)

32. Liu, J., Wu, B., Niu, Q.: Nonlinear evolution of quantum states in the adiabatic regime. Physical review letters 90(17), 170404 (2003) 